\title{
Large enhancement of Förster resonance energy transfer on graphene platforms
}

\author{
S.-A. Biehs ${ }^{1, a)}$ and G. S. Agarwal ${ }^{2}$ \\ ${ }^{1}$ Institut für Physik, Carl von Ossietzky Universität, D-26111 Oldenburg, Germany \\ ${ }^{2}$ Department of Physics, Oklahoma State University, Stillwater, Oklahoma 74078, USA
}

(Received 22 October 2013; accepted 25 November 2013; published online 12 December 2013)

\begin{abstract}
In view of the applications of Förster resonant energy transfer (FRET) in biological systems which especially require FRET in the infrared region, we investigate the great advantage of graphene plasmonics in such studies. Focusing on the fundamental aspects of FRET between a donoracceptor pair on a graphene platform showing that FRET mediated by the plasmons in graphene is broadband and enhanced by six orders of magnitude. We briefly discuss the impact of phononpolaritonic substrates. (C) 2013 AIP Publishing LLC. [http://dx.doi.org/10.1063/1.4847676]
\end{abstract}

Metal plasmonics has been investigated very intensively in the last decades. It is nowadays well known that in the visible metal structures like metal films or nanoparticles, for instance, are the plasmonic structures of choice exhibiting strong field confinement and large field enhancement close to the metallic structure. In contrast, research on graphene plasmonics which is in some sense complementary to metal plasmonics ${ }^{1}$ has just begun. The plasmons in graphene show strong field confinement in the infrared, whereas in the visible graphene plasmonics is challenging. ${ }^{2-5}$ One of the main advantages of using graphene in plasmonics or graphenebased hybrid-plasmonic devices is its tunability by doping or gating. ${ }^{6}$ Especially for applications of the Förster resonance energy transfer (FRET) such as in vivo infrared fluorescence imaging applications as for example lymph-node mapping and cancer imaging, ${ }^{7,8}$ graphene plasmonics is highly suitable and can unfold its full strength.

The FRET itself is widely used in biochemistry to study protein and RNA folding, ${ }^{9}$ DNA nanomechanical devices, ${ }^{10}$ and even to transport energy along a DNA backbone ${ }^{11}$ to mention a few applications. Theoretically, it was already shown in the 80s that in the vicinity of nanoparticles FRET between a donor (D) and an acceptor (A) molecule can be enhanced by two to five orders of magnitude in the idealized case when both the D and A are in resonance with the localized plasmon resonance. ${ }^{12,13}$ Recent theoretical works have also studied the impact of nonlocal effects and the possibility of magneto-optical control of FRET close to metallic nanoparticles. ${ }^{14,15}$ Although the plasmonic enhancement (PE) effect using nanoparticles could be measured lately, ${ }^{16,17}$ only moderate enhancement factors (EFs) of 3.5 and 8.6 were found. ${ }^{17}$ More recent experiments report FRET enhancements by a factor of $173 .{ }^{18}$ The PE could also be demonstrated experimentally for FRET between a D and A separated by a metal film. ${ }^{19}$

In this Letter, we demonstrate how graphene plasmonics can enhance FRET in the infrared. We want to emphasize that most recent studies are considering spontaneous emission close to graphene ${ }^{3,20-23}$ which is a first-order process, whereas FRET is a second-order process. $^{24} \mathrm{We}$ study in

\footnotetext{
a)biehs@theorie.physik.uni-oldenburg.de
}

detail the FRET between a D-A pair in close vicinity to a graphene sheet as depicted in Fig. 1. We show that FRET mediated by the plasmons in the graphene sheet is broadband and can be enhanced by six orders of magnitude. The broadband property of the PE is very advantageous since one can expect large PE also in cases where the emission and absorption spectra of the D and A do not fully overlap. Further, we show that the presence of graphene allows for energy transfer between perpendicularly oriented dipole moments which do not couple in free space. Finally, we discuss how the interaction of the graphene plasmons with the surface phonon polaritons of a SiC substrate affects the energy transfer. Since graphene is highly tunable this PE effect can be easily controlled even dynamically.

Using second-order perturbation theory, the energy transfer rate $\Gamma$ for dipole-dipole interaction between a D and an $\mathrm{A}$ in the presence of arbitrarily shaped, dispersing and absorbing bodies can be derived within the framework of macroscopic $\mathrm{QED}^{25}$ yielding

$$
\Gamma=\int \mathrm{d} \omega \sigma^{\mathrm{abs}}(\omega) T(\omega) \sigma^{\mathrm{em}}(\omega),
$$

where $\sigma^{\mathrm{abs}}$ and $\sigma^{\mathrm{em}}$ are the free space absorption and emission spectra of the A and the D. The energy transfer function $T(\omega)$ between the $\mathrm{D}$ and $\mathrm{A}$ is ${ }^{25}$

$$
T(\omega)=\frac{2 \pi}{\hbar^{2}}\left(\frac{\omega^{2}}{\epsilon_{0} c^{2}}\right)^{2}\left|\mathbf{d}_{\mathrm{D}}\right|^{2}\left|\mathbf{d}_{\mathrm{A}}\right|^{2}\left|\mathbf{e}_{\mathrm{A}} \cdot \mathbb{G}\left(\mathbf{r}_{A}, \mathbf{r}_{D}\right) \cdot \mathbf{e}_{\mathrm{D}}\right|^{2},
$$

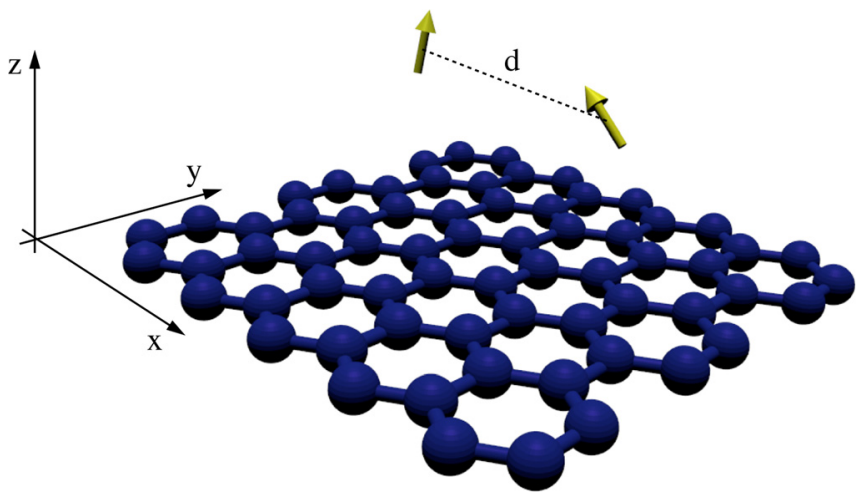

FIG. 1. Sketch of the D and A above a sheet of graphene. 
introducing the dipole-transition matrix elements $\mathbf{d}_{\mathrm{D}}$ $=\left|\mathbf{d}_{\mathrm{D}}\right| \mathbf{e}_{\mathrm{D}}$ and $\mathbf{d}_{\mathrm{A}}=\left|\mathbf{d}_{\mathrm{A}}\right| \mathbf{e}_{\mathrm{A}}$ of the $\mathrm{D}$ and $\mathrm{A}$. The unit vectors $\mathbf{e}_{\mathrm{D}}$ and $\mathbf{e}_{\mathrm{A}}$ determine the orientation of the dipole moments. $\epsilon_{0}$ is the permittivity of vacuum. Through the Green's function $\mathbb{G}\left(\mathbf{r}_{A}, \mathbf{r}_{D}\right)$ the environment of the $\mathrm{D}$ at $\mathbf{r}_{D}$ and the $\mathrm{A}$ at $\mathbf{r}_{A}$ is fully taken into account. The energy transfer function $T(\omega)$ can also be expressed in terms of the free space spontaneous emission rates ${ }^{26} \gamma_{D / A}=\omega^{3}\left|\mathbf{d}_{D / A}\right| / 3 \pi \epsilon_{0} \hbar c^{3}$. We obtain

$$
T(\omega)=18 \pi^{3} \gamma_{A} \gamma_{D} \frac{\left|\mathbf{e}_{\mathrm{A}} \cdot \mathbb{G}\left(\mathbf{r}_{A}, \mathbf{r}_{D}\right) \cdot \mathbf{e}_{\mathrm{D}}\right|^{2}}{(2 \pi / \lambda)^{2}} .
$$

By normalizing the emission and absorption spectra by the free space emission rates $\tilde{\sigma}^{\mathrm{em}} \equiv \sigma^{\mathrm{em}} \gamma_{D}$ and $\tilde{\sigma}^{\mathrm{abs}} \equiv \sigma^{\mathrm{abs}} \gamma_{A}$ we can rewrite the expression for the energy transfer as

$$
\Gamma=\int \mathrm{d} \omega \tilde{\sigma}^{\mathrm{abs}}(\omega) \tilde{T}(\omega) \tilde{\sigma}^{\mathrm{em}}(\omega),
$$

introducing the dimensionless energy transfer function

$$
\tilde{T}(\omega) \equiv 18 \pi^{3} \frac{\left|\mathbf{e}_{\mathrm{A}} \cdot \mathbb{G}\left(\mathbf{r}_{A}, \mathbf{r}_{D}\right) \cdot \mathbf{e}_{\mathrm{D}}\right|^{2}}{(2 \pi / \lambda)^{2}} .
$$

The EF for the energy transfer close to a plasmonic structure like graphene in comparison to vacuum is defined as

$$
E(\omega) \equiv \frac{\tilde{T}(\omega)}{\tilde{T}^{(\mathrm{vac})}(\omega)}=\frac{\left|\mathbf{e}_{\mathrm{A}} \cdot \mathbb{G} \cdot \mathbf{e}_{\mathrm{D}}\right|^{2}}{\left|\mathbf{e}_{\mathrm{A}} \cdot \mathbb{G}^{(\mathrm{vac})} \cdot \mathbf{e}_{\mathrm{D}}\right|^{2}},
$$

where the Green's function $\mathbb{G}=\mathbb{G}^{(\mathrm{vac})}+\mathbb{G}^{(\mathrm{sc})}$ is a sum of the vacuum Green's function $\mathbb{G}^{\text {(vac) }}$ and the scattered Green's function $\mathbb{G}^{(\mathrm{sc})}$. The explicit expressions of the Green's functions for free space and planar structures can be found in Refs. 26 and 28 (see also Ref. 27).

Before we can determine the energy transfer rate between the D and A close to a sheet of graphene it is necessary to determine its optical properties which enter in the scattered Green's function. The optical properties of graphene are fully described by its in-plane conductivity $\sigma=\sigma_{D}+\sigma_{I}$ consisting of the Drude-like intraband contribution $\sigma_{D}$ and the interband contribution $\sigma_{I}$ which are given by ${ }^{29}$

$$
\begin{gathered}
\sigma_{D}=\frac{\mathrm{i}}{\omega+\mathrm{i} / \tau} \frac{2 e^{2} K_{\mathrm{B}} T}{\pi \hbar^{2}} \log \left[2 \cosh \left(\frac{E_{F}}{2 K_{\mathrm{B}} T}\right)\right] \\
\sigma_{I}=\frac{e^{2}}{4 \hbar}\left[G\left(\frac{\hbar \omega}{2}\right)+\mathrm{i} \frac{4 \hbar \omega}{\pi} \int_{0}^{\infty} \mathrm{d} \xi \frac{G(\xi)-G(\hbar \omega / 2)}{(\hbar \omega)^{2}-4 \xi^{2}}\right],
\end{gathered}
$$

where $G(x)=\sinh \left(x / K_{\mathrm{B}} T\right) /\left[\cosh \left(E_{F} / K_{\mathrm{B}} T\right)+\cosh \left(x / K_{\mathrm{B}} T\right)\right]$. Here $T$ is the temperature of the graphene sheet, $E_{F}$ is its Fermi-level which can be tuned by gating and doping for instance. $\tau$ is a phenomenological damping constant, $K_{\mathrm{B}}$ is Boltzmann's constant, $2 \pi \hbar$ is Planck's constant, and $e$ is the electron charge. The in-plane conductivity enters in the Fresnel reflection coefficients. For a graphene sheet on a dielectric substrate with permittivity $\epsilon$, the reflection coefficients for s- and p-polarized light are given by ${ }^{3,30}$ (see also Ref. 27)

$$
r_{\mathrm{s}}=\frac{\gamma_{0}-\gamma-\sigma \omega}{\gamma_{0}+\gamma+\sigma \omega} \quad \text { and } \quad r_{\mathrm{p}}=\frac{\epsilon \gamma_{0}-\gamma+\frac{\sigma \gamma \gamma_{0}}{\omega \epsilon_{0}}}{\epsilon \gamma_{0}+\gamma+\frac{\sigma \gamma \gamma_{0}}{\omega \epsilon_{0}}}
$$

where $\kappa=\left(k_{x}, k_{y}\right)^{t}$ is the in-plane wave vector and $\gamma$ $=\sqrt{k_{0}^{2} \epsilon-\kappa^{2}}$ and $\gamma_{0}=\sqrt{k_{0}^{2}-\kappa^{2}}$ are the out-of-plane wave vectors inside the substrate and in vacuum. The substrate can have quite a large impact on the properties of the plasmons in graphene, in particular, when it supports surface phonon polaritons like $\mathrm{SiO}_{2}$ and $\mathrm{SiC} .^{23,31,32}$ For convenience, we will neglect the influence of the substrate in the following and focus mainly on suspended graphene.

The plasmons in graphene propagating along the sheet are determined by the poles of the reflection coefficient for $\mathrm{p}$ polarization. When assuming that we have suspended graphene $(\epsilon=1)$, then the dispersion relation of the plasmon simply reads

$$
\kappa_{\mathrm{P}}=\sqrt{k_{0}^{2}-\left(\frac{2 \omega \epsilon_{0}}{\sigma}\right)^{2}}
$$

From this dispersion relation, one can easily determine the wavelength $\lambda_{\mathrm{P}}=2 \pi / \operatorname{Re}\left(\kappa_{\mathrm{P}}\right)$ and the propagation length $l_{\mathrm{P}}=1 / \operatorname{Im}\left(\kappa_{\mathrm{P}}\right)$ of the plasmon. For a relatively high Fermi level $E_{\mathrm{F}}=1 \mathrm{eV}$ which can be realized nowadays, ${ }^{6}$ Table I summarizes some of the plasmon properties as the in-plane confinement and the propagation length for frequencies ranging from $0.2-0.9 \mathrm{eV}$. The in-plane confinement $\lambda_{\mathrm{P}} / \lambda$ is in this case strong and can attain values of 0.013 to 0.072 . The out-of-plane confinement is even stronger: since the out-ofplane wave vector is approximately $\mathrm{i} \kappa_{\mathrm{P}}$, the out-of-plane confinement is given by $1 / \operatorname{Re}\left(\kappa_{\mathrm{P}}\right)=\lambda_{\mathrm{P}} /(2 \pi)$. This means that the out-of-plane confinement is between $0.002 \lambda$ and $0.011 \lambda$. The propagation length $l_{\mathrm{P}}$ of the plasmons in graphene is found to be between $300 \mathrm{~nm}$ and $2 \mu \mathrm{m}$ depending on the frequency so that $l_{\mathrm{P}} / \lambda_{\mathrm{P}}$ is between 17.4 and 4.8 . Note that the propagation length approximately scales linearly with the relaxation time $\tau$ so that $l_{\mathrm{P}} / \lambda_{\mathrm{P}}$ can even reach values on the order of 100 for $\tau=10^{-12} \mathrm{~s}^{-1}$ as shown in Ref. 3. Further note that the plasmon properties can be easily tuned by changing the Fermi level by gating or tuning.

In order to demonstrate the PE, we consider a D and an A on a plane parallel to the graphene sheet. The distance of this plane to the sheet of graphene which is assumed to be in the $\mathrm{x}-\mathrm{y}$ plane is chosen to be $z=10 \mathrm{~nm}$. In Figs. 2(a)-2(c), the EF $E$ defined in Eq. (6) is plotted as a function of distance $d$ between the $\mathrm{D}$ and the A for parallely aligned dipole

TABLE I. The in-plane confinement $\lambda_{\mathrm{P}} / \lambda$, the propagation length $l_{\mathrm{P}}$, and $l_{\mathrm{P}} / \lambda_{\mathrm{P}}$ of the surface plasmons in graphene using $T=300 \mathrm{~K}, E_{F}=1 \mathrm{eV}$ and $\tau=10^{-13} \mathrm{~s}^{-1}$

\begin{tabular}{lcccc}
\hline \hline Frequency $(\mathrm{eV})$ & $\lambda(\mu \mathrm{m})$ & $\lambda_{\mathrm{P}} / \lambda$ & $l_{\mathrm{P}}(\mathrm{nm})$ & $l_{\mathrm{P}} / \lambda_{\mathrm{P}}$ \\
\hline 0.2 & 6.23 & 0.072 & 2142 & 4.8 \\
0.3 & 4.15 & 0.047 & 1397 & 7.08 \\
0.5 & 2.49 & 0.027 & 773 & 11.3 \\
0.7 & 1.78 & 0.018 & 486 & 14.9 \\
0.9 & 1.38 & 0.013 & 311 & 17.4 \\
\hline \hline
\end{tabular}



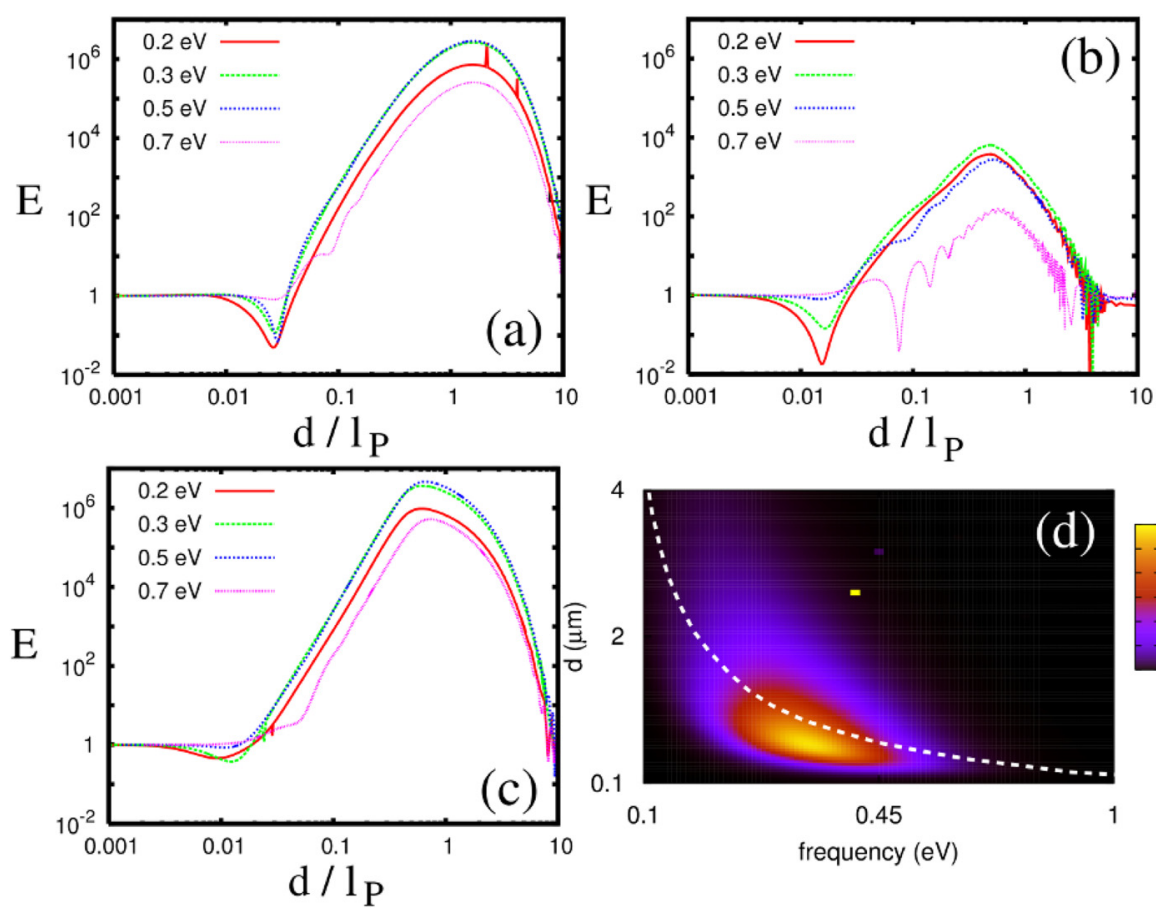

$\mathrm{E}$

(a)

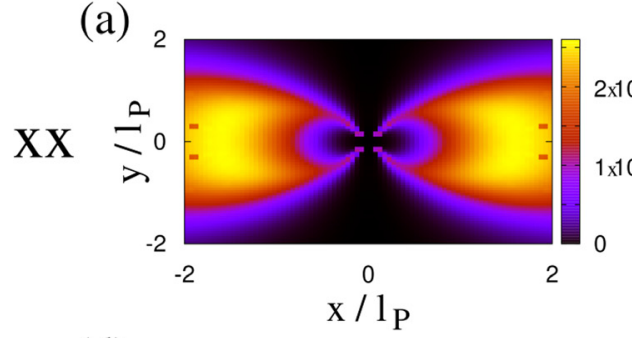

(d)

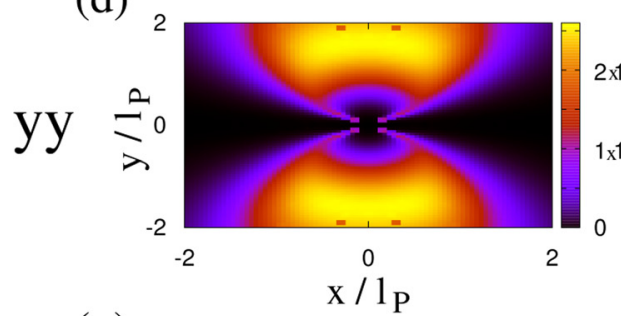

(g)

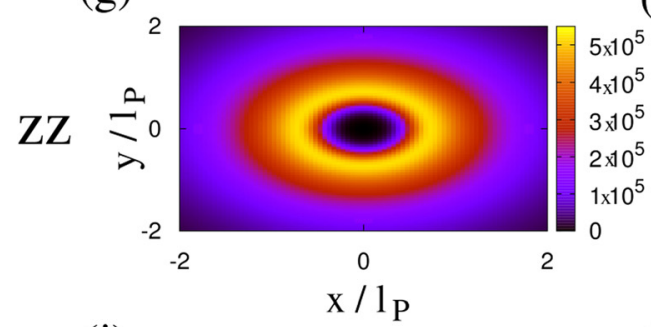

(j)

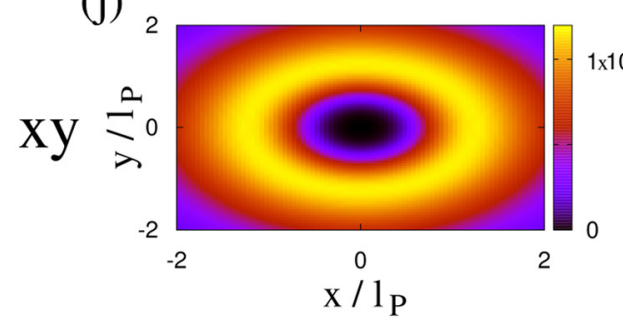

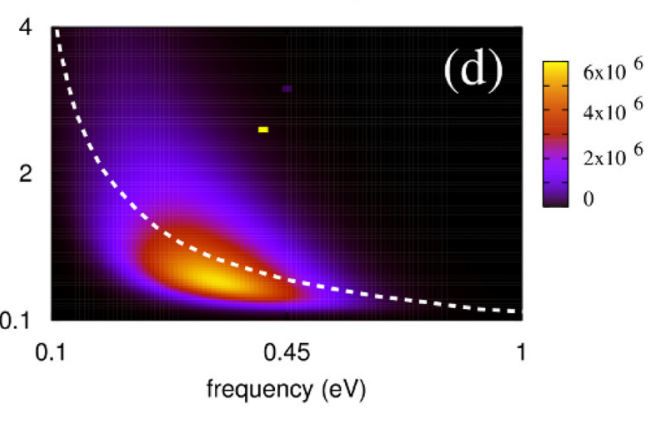

$\widetilde{\mathrm{T}}$

(b)

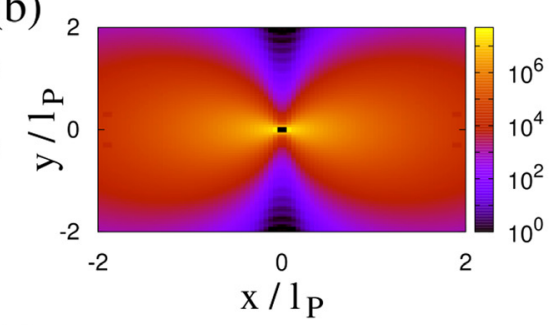

(e)

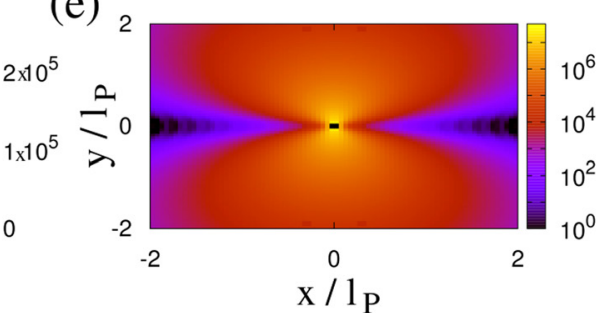

(h)

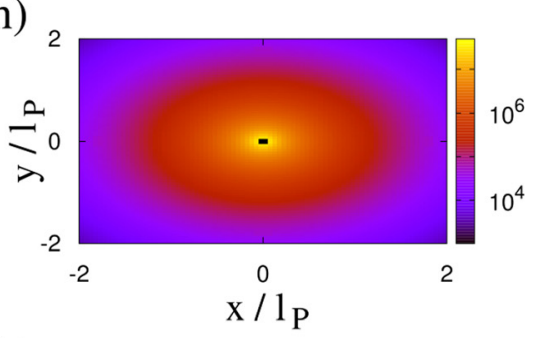

(k)

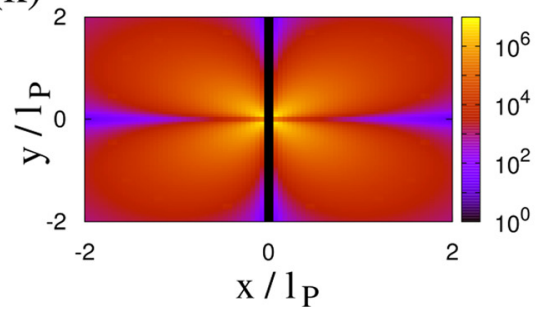

FIG. 2. (a)-(c) Enhancement factor $E$ for $\mathrm{D}$ and $\mathrm{A}$ placed along the $\mathrm{X}$-axis as a function of distance $d$ for different frequencies ranging from 0.2 to $0.7 \mathrm{eV}$ $\left(z=10 \mathrm{~nm}\right.$ and $\left.E_{F}=1 \mathrm{eV}\right)$. The distance is for each frequency normalized to the corresponding propagation length $l_{\mathrm{P}}$ of the graphene plasmon. The orientations of the dipole moment are (a) $\mathbf{e}_{\mathrm{A}}=\mathbf{e}_{\mathrm{D}}=\mathbf{e}_{x}$, (b) $\mathbf{e}_{\mathrm{A}}=\mathbf{e}_{\mathrm{D}}=\mathbf{e}_{y}$, and (c) $\mathbf{e}_{\mathrm{A}}=\mathbf{e}_{\mathrm{D}}=\mathbf{e}_{z}$. (d) Enhancement factor $E$ for $\mathbf{e}_{\mathrm{A}}=\mathbf{e}_{\mathrm{D}}=\mathbf{e}_{z}$ as a function of frequency and distance. The white dashed line is a plot of $1 / \operatorname{Im}\left(\kappa_{\mathrm{P}}\right)$ from Eq. (10).
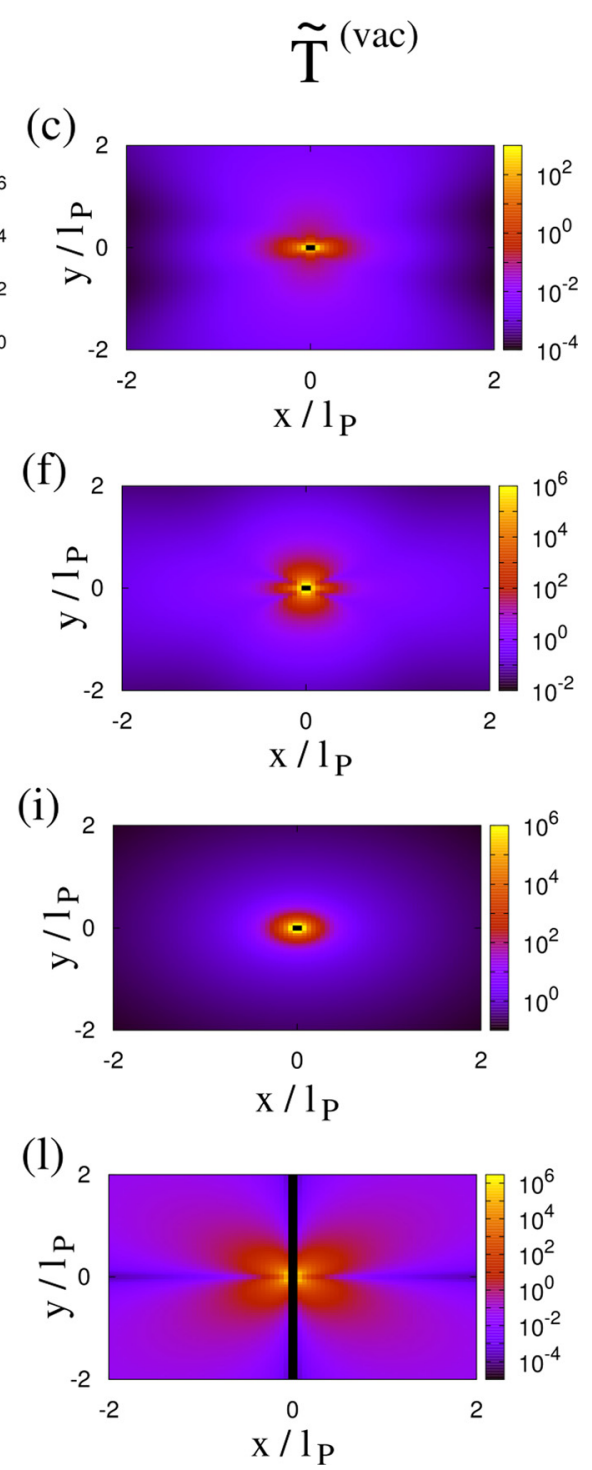

FIG. 3. Enhancement factor $E$ (first column), the energy transfer function close to graphene $\tilde{T}$ (second column) and in free space $\tilde{T}^{(\text {vac) }}$ (third column) in the $\mathrm{x}$ y plane for $\omega=0.7 \mathrm{eV}$ and (a)-(c) $\mathbf{e}_{\mathrm{A}}=\mathbf{e}_{\mathrm{D}}=\mathbf{e}_{x}$, (d)-(f) $\mathbf{e}_{\mathrm{A}}=\mathbf{e}_{\mathrm{D}}=\mathbf{e}_{y}$, (g)-(i) $\mathbf{e}_{\mathrm{A}}=\mathbf{e}_{\mathrm{D}}=\mathbf{e}_{z}$, and (j) $-(\mathrm{l}) \mathbf{e}_{\mathrm{A}}=\mathbf{e}_{x}$ and $\mathbf{e}_{\mathrm{D}}=\mathbf{e}_{y}$. The $\mathrm{x}$ and $\mathrm{y}$ coordinates are normalized to the propagation length of the graphene plasmon $l_{\mathrm{P}}=486 \mathrm{~nm}, z=10 \mathrm{~nm}$, and $E_{F}=1 \mathrm{eV}$. 

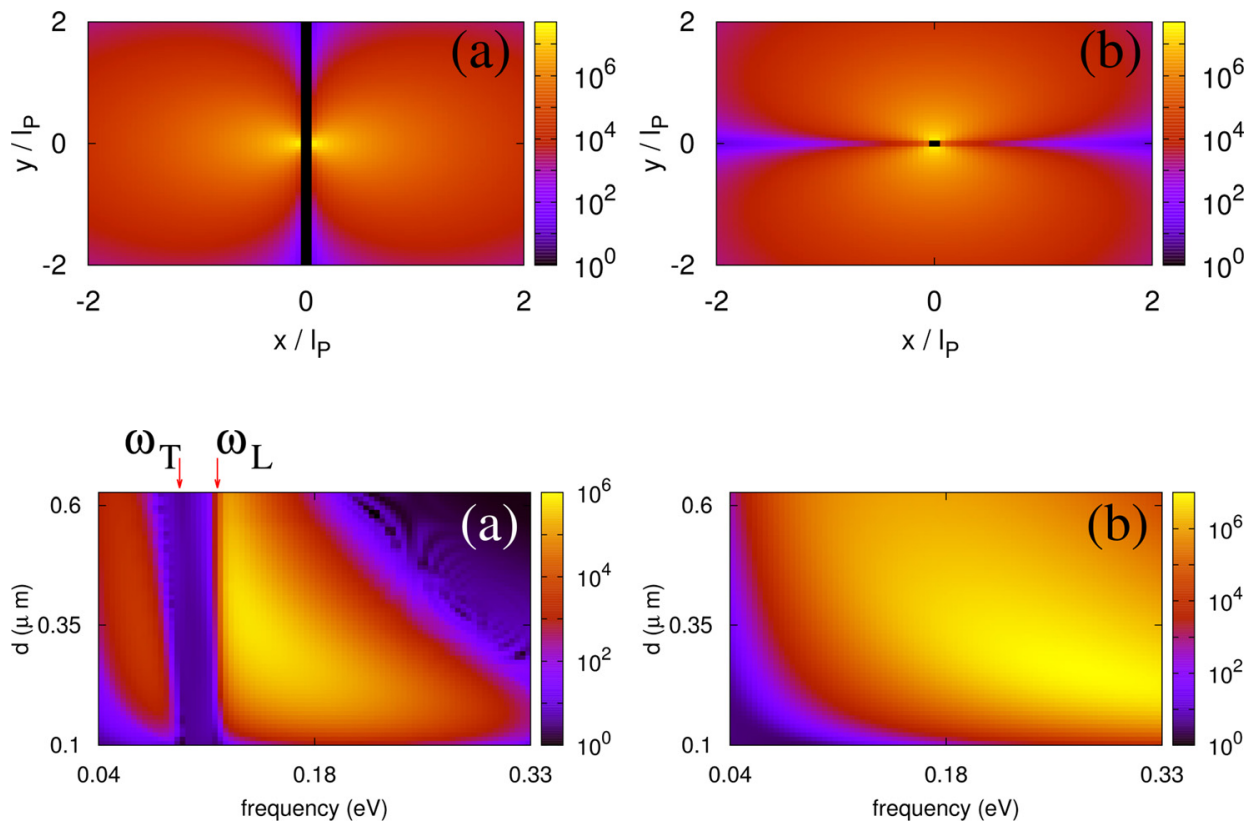

FIG. 4. Energy transfer function $\tilde{T}$ for (a) $\mathbf{e}_{\mathrm{A}}=\mathbf{e}_{x}$ and $\mathbf{e}_{\mathrm{D}}=\mathbf{e}_{z}$ and (b) $\mathbf{e}_{\mathrm{A}}=\mathbf{e}_{y}$ and $\mathbf{e}_{\mathrm{D}}=\mathbf{e}_{z}$ in $\mathrm{x}-\mathrm{y}$ plane. The $\mathrm{x}$ and $\mathrm{y}$ axis are normalized to propagation length $l_{\mathrm{P}}, z=10 \mathrm{~nm}, E_{F}=1 \mathrm{eV}$, and $\omega=0.7 \mathrm{eV}$. moments along the $x, y$, and $z$ axis. It can be seen that FRET mediated by the plasmons in graphene can be six orders of magnitude larger than in free space. That means that also the recently studied nonparaxial spin-Hall effect of light ${ }^{33}$ will be extraordinary large in the vicinity of graphene. Further, the maximum enhancement is obtained for distances on the order of the propagation length of the graphene plasmons. In Fig. 2(d) we show a plot of the EF $E$ as a function of distance $d$ and frequency $\omega$ for the case where the dipole moments of the $\mathrm{D}$ and $\mathrm{A}$ are oriented along the $\mathrm{z}$ axis. It becomes apparent that the PE is broadband in frequency for distances $d$ between $100 \mathrm{~nm}$ and $1 \mu \mathrm{m}$. Hence, one can expect that the predicted large enhancement of FRET can be achieved in the realistic case where the emission and absorption spectra of the $\mathrm{D}$ and $\mathrm{A}$ are nonresonant in contrast to the case of metallic nanoparticles where the resonance of the localized plasmon is narrow band.

In Figs. 3(a), 3(d), 3(g), and 3(j) we show plots of $E$ where the $A$ is placed at $\mathbf{r}_{A}=(0 \mathrm{~nm}, 0 \mathrm{~nm}, 10 \mathrm{~nm})$ and the $\mathrm{x}-\mathrm{y}$ position of the $\mathrm{D}$ position $\mathbf{r}_{D}=(x, y, 10 \mathrm{~nm})$ is varied. Once more it becomes obvious that the largest EFs can be obtained for distances around the propagation length of the graphene plasmons $l_{\mathrm{P}}$. In addition, we have plotted the energy transfer function above graphene $\tilde{T}$ and in free space $\tilde{T}^{(\text {vac) }}$. Note that the EF $E$ is invariant under rotation around the $z$ axis for $\mathbf{e}_{\mathrm{A}}=\mathbf{e}_{x}$ and $\mathbf{e}_{\mathrm{D}}=\mathbf{e}_{y}$ although $\tilde{T}$ and $\tilde{T}^{(\mathrm{vac})}$ are not.

It is interesting to note that due to the presence of the graphene sheet there is also FRET between the D and A for $\left(\mathbf{e}_{\mathrm{A}}=\mathbf{e}_{x}, \mathbf{e}_{\mathrm{D}}=\mathbf{e}_{z}\right)$ and $\left(\mathbf{e}_{\mathrm{A}}=\mathbf{e}_{y}, \mathbf{e}_{\mathrm{D}}=\mathbf{e}_{z}\right)$. The free space FRET vanishes in this case so that $E$ is not a well defined quantity for such orientations of dipole moments. But the energy transfer function $\tilde{T}(\omega)$ shown in Fig. 4 indicates that the PE of FRET is in the same order of magnitude as for the cases considered in Fig. 3.

Finally, we briefly discuss the impact of the substrate. In Fig. 5(a) we show a plot of the EF $E$ of FRET above a graphene sheet deposited on $\mathrm{SiC}$ as a function of frequency and distance. We choose $z=10 \mathrm{~nm}$ and $E_{F}=0.5 \mathrm{eV}$. For comparison we also plot the EF for suspended graphene in Fig. 5(b) using the same parameters (for similar plots using $E_{F}=1 \mathrm{eV}$ see supplementary material ${ }^{27}$ ). In contrast to the results found in Fig. 5(b) we can see a gap of negligible small FRET in the reststrahlen region of $\mathrm{SiC}$ (i.e., frequencies between the transversal and longitudinal phonon frequencies $\omega_{\mathrm{T}}$ and $\left.\omega_{\mathrm{L}}\right) .{ }^{23,31}$ In addition, due to the interaction of the graphene plasmon with the surface-phonon polaritons in $\mathrm{SiC}$ the $\mathrm{PE}$ is redshifted and concentrated at frequencies slightly larger than $\omega_{\mathrm{L}}=0.12 \mathrm{eV}$. It can also be seen that the EF on graphene/SiC is slightly smaller and the effect is more narrowband compared to the case of suspended graphene. Hence, by choosing an active or inactive substrate the observed FRET enhancement can be further controlled.

In summary, we have shown that in close vicinity to a sheet of graphene FRET can be enhanced by six orders of magnitude. The maximum enhancement is obtained for D-A distances on the order of the propagation length of the plasmons in graphene. Further, we have shown that the enhancement effect is broadband and depends not only on the relative position of the $\mathrm{D}$ and $\mathrm{A}$ but also on the orientation of their dipole moments. In particular, the presence of graphene allows for coupling of perpendicularly oriented dipole moments which do not couple in free space. The role of the substrate is briefly discussed using SiC. Hence, the observed plasmonic enhancement can be tuned by doping or gating of graphene and by choosing an appropriate substrate.

${ }^{1}$ F. Xia, Nat. Photonics 7, 420 (2013).

${ }^{2}$ M. Jablan, H. Buljan, and M. Soljacic, Phys. Rev. B 80, 245435 (2009).

${ }^{3}$ F. H. L. Koppens, D. E. Chang, and F. J. Garcia de Abajo, Nano Lett. 11, 3370 (2011).

${ }^{4}$ V. W. Brar, M. S. Jang, M. Sherrott, J. J. Lopez, and H. A. Atwater, Nano Lett. 13, 2541 (2013).

${ }^{5}$ Yu. V. Bludov, A. Ferreira, N. M. R. Peres, and M. I. Vasilevskiy, Int. J. Mod. Phys. B 27, 1341001 (2013).

${ }^{6}$ A. N. Grigorenko, M. Polini, and K. S. Novoselov, Nat. Photonics 6, 749 (2012).

${ }^{7}$ X. He, Y. Wang, K. Wang, M. Chen, and S. Chen, Anal. Chem. 84, 9056 (2012).

${ }^{8}$ L. Xiong, A. J. Shuhendler, and J. Rao, Nat. Commun. 3, 1193 (2012).

${ }^{9}$ S. Weiss, Nat. Struct. Biol. 7, 724 (2000). 
${ }^{10}$ B. K. Müller, A. Reuter, F. C. Simmel, and D. C. Lamb, Nano Lett. 6, 2814 (2006).

${ }^{11}$ S. Vyawahare, S. Eyal, K. D. Mathews, and S. R. Quake, Nano Lett. 4, 1035 (2004).

${ }^{12}$ J. I. Gersten and A. Nitzan, Chem. Phys. Lett. 104, 31 (1984).

${ }^{13}$ X. M. Hua, J. I. Gersten, and A. Nitzan, J. Chem. Phys. 83, 3650 (1985).

${ }^{14}$ H. Y. Xie, H. Y. Chung, P. T. Leung, and D. P. Tsai, Phys. Rev. B 80, 155448 (2009).

${ }^{15}$ R. Vincent and R. Carminati, Phys. Rev. B 83, 165426 (2011).

${ }^{16}$ J. Malicka, I. Gryczynski, J. Fang, J. Kusba, and J. R. Lakowicz, Anal. Biochem. 315, 160 (2003).

${ }^{17}$ F. Reil, U. Hohenester, J. R. Krenn, and A. Leitner, Nano Lett. 8, 4128 (2008).

${ }^{18}$ M. L. Viger, D. Brouard, and D. Boudreau, J. Phys. Chem. C 115, 2974 (2011).

${ }^{19}$ P. Andrew and W. L. Barnes, Science 306, 1002 (2004).

${ }^{20}$ K. A. Velizhanin and A. Emifov, Phys. Rev. B 84, 085401 (2011).

${ }^{21}$ G. Gómez-Santos and T. Stauber, Phys. Rev. B 84, 165438 (2011).

${ }^{22}$ J. Tisler, T. Oeckinghaus, R. J. Stöhr, R. Kolesov, R. Reuter, F. Reinhardt, and J. Wrachtrup, Nano Lett. 13, 3152 (2013).
${ }^{23}$ R. Messina, J.-P. Hugonin, J.-J. Greffet, F. Marquier, Y. De Wilde, A. Belarouci, L. Frechette, Y. Cordier, and P. Ben-Abdallah, Phys. Rev. B 87, 085421 (2013).

${ }^{24}$ G. S. Agarwal, Quantum Optics (Cambridge University Press, Cambridge, 2012).

${ }^{25}$ H. T. Dung, L. Knöll, and D.-G. Welsch, Phys. Rev. A 65, 043813 (2002).

${ }^{26}$ L. Novotny and B. Hecht, Principles of Nano-Optics (Cambridge University Press, Cambridge, 2006).

${ }^{27}$ See supplementary material at http://dx.doi.org/10.1063/1.4847676 for the detailed expressions for the Green's tensor, for a brief discussion of the material properties of graphene, and a plot of the enhancement factor for graphene on $\mathrm{SiC}$ in comparison to suspended graphene.

${ }^{28}$ J. E. Sipe, J. Opt. Soc. Am. B 4, 481 (1987).

${ }^{29}$ L. A. Falkovsky and A. A. Varlamov, Eur. Phys. J. B 56, 281 (2007).

${ }^{30}$ T. Stauber, N. M. R. Peres, and A. K. Geim, Phys. Rev. B 78, 085432 (2008).

${ }^{31}$ R. J. Koch, Th. Seyller, and J. A. Schaefer, Phys. Rev. B 82, 201413(R) (2010).

${ }^{32}$ H. Yan, T. Low, W. Zhu, Y. Wu, M. Freitag, X. Li, F. Guinea, P. Avouris, and F. Xia, Nat. Photonics 7, 394 (2013).

${ }^{33}$ G. S. Agarwal and S.-A. Biehs, Opt. Lett. 38, 4421 (2013). 